\title{
A Rare Case of Renal AA Amyloidosis Secondary to Sjogren's Syndrome
}

\author{
Mylene Costa $^{1}$, Helena Greenfield ${ }^{1}$, Ricardo Pereira $^{1}$, Teresa Chuva $^{2}$, Rui Henrique $^{3}$, Cátia Cunha $^{4}$ \\ ${ }^{1}$ Internal Medicine, Hospital Pedro Hispano, Porto, Portugal \\ ${ }^{2}$ Nephrology, Instituto Português de Oncologia do Porto, Portugal \\ ${ }^{3}$ Pathological Anatomy, Instituto Português de Oncologia do Porto, Portugal \\ ${ }^{4}$ Nephrology, Hospital Pedro Hispano, Porto, Portugal
}

Received: 22/07/2019

Accepted: $12 / 08 / 2019$

Published: $16 / 09 / 2019$

How to cite this article: Costa M, Greenfield H, Pereira R, Chuva T, Henrique R, Cunha C. A rare case of renal AA amyloidosis secondary to Sjorgen's syndrome. EJCRIM 2019;6: doi:10.12890/2019_001226.

Conflicts of Interests: The Authors declare that there are no competing interest

Acknowledgements: The authors gratefully acknowledge Dr. Helena Greenfield (Internal Medicine, Hospital Pedro Hispano, Portugal) for her help in writing and translating the manuscript and Dr. Teresa Chuva (Nephrology, Instituto Português de Oncologia do Porto, Portugal) for providing the pathology images for this article.

This article is licensed under a Commons Attribution Non-Commercial 4.0 License

\section{ABSTRACT}

AA amyloidosis is a rare complication of chronic inflammatory disorders and has been associated with rheumatoid arthritis and ankylosing spondylitis.

We present a case of AA amyloidosis secondary to Sjogren's syndrome (SS). A 79-year-old woman presented with rapidly progressive renal failure and complaints of asthenia, anorexia and generalized oedema. She had severe renal failure (creatinine $6.0 \mathrm{mg} / \mathrm{dl}$ ), with microscopic haematuria, nephrotic proteinuria and low serum albumin levels, and an increased erythrocyte sedimentation rate. Serum protein electrophoresis revealed a peak in the gamma globulin zone. The patient was started on haemodialysis and corticosteroids. Clinical results showed the patient met the diagnostic criteria for primary SS, and neoplastic haematological disease was excluded. Renal biopsy revealed a diagnosis of $A A$ amyloidosis.

Renal AA amyloidosis is a rare condition in patients with primary SS. However, in patients with proteinuria and/or renal failure, it should be included in the differential diagnosis and a renal biopsy should be performed.

\section{LEARNING POINTS}

- Sjogren's syndrome should be regarded as a predisposing condition for the development of renal AA amyloidosis.

- Sjogren's syndrome and renal AA amyloidosis have been diagnosed simultaneously in some patients.

- A renal biopsy should be performed in patients with Sjogren's syndrome and proteinuria and/or renal failure.

\section{KEYWORDS}

Sjogren's syndrome, AA amyloidosis, renal amyloidosis, renal biopsy

\section{INTRODUCTION}

Primary Sjogren's syndrome (SS) is a chronic autoimmune disorder characterized by lymphocytic infiltration of exocrine glands and extraglandular sites ${ }^{[1-4]}$. The incidence of renal involvement in SS is $<10 \%{ }^{[2]}$.

Different types of renal involvement are described, most frequently tubulointerstitial nephritis ( 60-75\%) and less frequently, glomerular nephritis (non-epithelial, secondary to immune complex deposition) (5-30\%) ${ }^{[1-4]}$. On the other hand, polyclonal activation of B-cells can induce cryoglobulinaemia and lead to the development of systemic vasculitis and membranoproliferative glomerulonephritis. 
Additionally, chronic B-cell activation is responsible for the more severe complication of primary SS (2-9\%) as a result of B-cell non-Hodgkin's lymphoma, which may rarely involve the kidney ${ }^{[4]}$. Although AA amyloidosis is associated with aetiologies such as rheumatoid arthritis and ankylosing spondylitis ${ }^{[5-9]}$, the association between SS and amyloidosis is not well established ${ }^{[8]}$.

\section{CASE REPORT}

We present the case of a 79-year-old woman, with hypertensive and valvular (moderate aortic stenosis) heart disease, who was admitted to the emergency room with complaints of asthenia, anorexia and generalized oedema associated with reduced urinary output.

She presented with acute renal failure (creatinine was $6.0 \mathrm{mg} / \mathrm{dl}$; it had been $0.99 \mathrm{mg} / \mathrm{dl} 1$ year before admission and $1.67 \mathrm{mg} / \mathrm{dl} 1 \mathrm{month}$ previously), microscopic haematuria (25-50 cells/field), nephrotic proteinuria (protein-to-creatinine ratio 3.5, albumin-to-creatinine ratio 9927 mg/g, proteinuria 7.2 g/24-hour urine), hypoalbuminaemia (2.2 mg/dl), hypercholesterolaemia, severe anaemia (haemoglobin 7.3 g/ $\mathrm{dl}$, normocytic/normochromic, no evidence of haemolysis, no iron, folates or vitamin B12 deficiency, and normal thyroid function), ESR 108 $\mathrm{mm} / \mathrm{h}$ and serum protein electrophoresis with a peak in the gamma globulin zone.

A thoraco-abdominal CT scan revealed pleural and pericardial effusion and excluded adenopathy and organomegaly, while abdominal ultrasonography showed normal-sized kidneys (right: $10.5 \mathrm{~cm}$ and left: $9.8 \mathrm{~cm}$ ) with loss of corticomedullary differentiation and no urological complications.

The patient was started on haemodialysis and on $40 \mathrm{mg}$ id oral dexamethasone, for 4 days, due to an initial suspicion of multiple myeloma. Subsequently, blood tests revealed speckled pattern 1:80 anti-nuclear antibodies (ANA), with cytoplasmatic dot staining, positive anti-SSA (>200 U/ml), anti-SSB $(40 \mathrm{U} / \mathrm{hl})$ and rheumatoid factor $(88.6 \mathrm{lU} / \mathrm{ml})$ with C3 $(54 \mathrm{mg} / \mathrm{dl})$ and C4 $(11.4 \mathrm{mg} / \mathrm{dl})$ consumption. The rest of the immunological study was negative (Table 1).

\begin{tabular}{|l|l|}
\hline Parameter & Result \\
\hline ANA & $1: 80$ \\
\hline Anti-SSA & $>200 \mathrm{U} / \mathrm{ml}$ (normal: $<7$ ) \\
Anti-SSB & $40 \mathrm{U} / \mathrm{nl}$ (normal: $<7$ ) \\
\hline C3 & $54 \mathrm{mg} / \mathrm{dl}$ (normal: 83-193) \\
C4 & $11.4 \mathrm{mg} / \mathrm{dl}$ (normal: 15-57) \\
\hline Rheumatoid factor & Positive \\
Anti-citrulline & Negative \\
\hline $\begin{array}{l}\text { Anti-RNP } \\
\text { Anti-Scl-70 }\end{array}$ & Negative \\
Anti-Jo-1 & \\
\hline Anti-centromere & \\
\hline Anti-dsDNA & Negative \\
\hline Anti-MPO & Negative \\
Anti-PR-3 & \\
\hline Immunoglobulin A, M and G & Normal \\
\hline IgG subtypes & Normal \\
\hline Lupus anticoagulant & Negative \\
Anticardiolipin lgG and lgM & \\
\hline Anti-beta2-glycoprotein & Negative \\
\hline Cryoglobulins & Negative \\
\hline ACE & \\
\hline HBs antigen, HIV, HCV & \\
\hline
\end{tabular}

Table 1. Immunology and serology diagnostic tests

Based on these results, oral prednisolone $1 \mathrm{mg} / \mathrm{kg}$ daily was initiated. Additional investigations included a positive Schirmer's test (left eye $1 \mathrm{~mm} / 5 \mathrm{~min}$; right eye $5 \mathrm{~mm} / 5 \mathrm{~min}$ ) and chronic grade 3 sialadenitis lesions (Chisholm and Mason classification) on salivary gland biopsy (Congo red staining was negative). Therefore, the patient met the 2016 ACR/EULAR criteria for SS.

As immunoelectrophoresis results showed IgG kappa monoclonal gammopathy in serum and urine, and there was increased B2-microglobulin of 28.25 (normal: 0.97-2.64) and elevated free kappa and lambda plasma light free chains (10.9/4.74, with an increased kappa/lambda ratio), 
the exclusion of neoplastic haematological disease through a myelogram ( $<1 \%$ of plasma cells) and immunophenotyping of peripheral blood (no lymphoproliferative disease) was crucial.

Finally, a renal biopsy was performed, which, under light microscopy, revealed a total of 16 glomeruli, of which 11 were globally sclerosed/ obsolete; in the remaining 5, mesangial expansion with a nodular and amorphous appearance was visible, without other glomerular changes (no proliferative changes or glomerular basement membrane alterations) (Fig. 1). The findings demonstrated 25-50\% tubulointerstitial fibrosis, with lymphocytic inflammatory infiltrate only in the areas of fibrosis (Fig. 2).
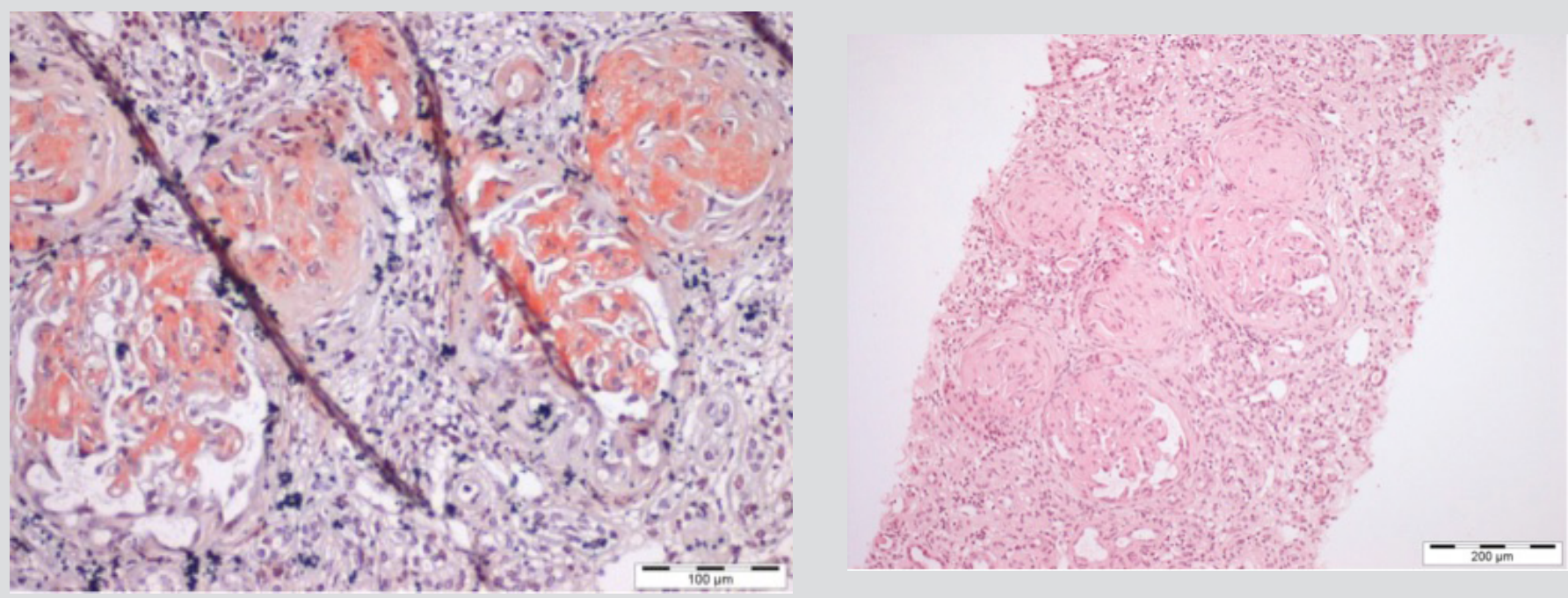

Figure 1. Amorphous, acellular glomerular deposits expanding the mesangial areas (haematoxylin and eosin staining)

Figure 2. Trichrome staining showing 25-50\% tubulointerstitial fibrosis, with inflammatory infiltrate in the areas of fibrosis

While awaiting the results of Congo red staining and immunofluorescence, the patient continued prednisolone $1 \mathrm{mg} / \mathrm{kg}$ daily and demonstrated symptomatic improvement. Volaemia was optimized and haemodynamic stability maintained, but without signs of renal function recovery, maintaining dialysis dependence. Unfortunately, on the 22th day of hospitalization, the patient experienced an unexpected and fatal cardiac arrest of unidentified cause. The patient's baseline electrocardiogram showed sinus rhythm, but, as previously mentioned, she had moderate aortic stenosis which is a known cause of sudden cardiac arrest.

Final renal biopsy results subsequently revealed Congo red-positive glomerular deposits (Fig. 3), while immunofluorescence was negative for IgG, IgM, IgA, C1q and C3. Immunohistochemistry revealed positivity for serum amyloid A protein.

Therefore, a diagnosis of renal secondary AA-type amyloidosis secondary to chronic SS inflammation was made.

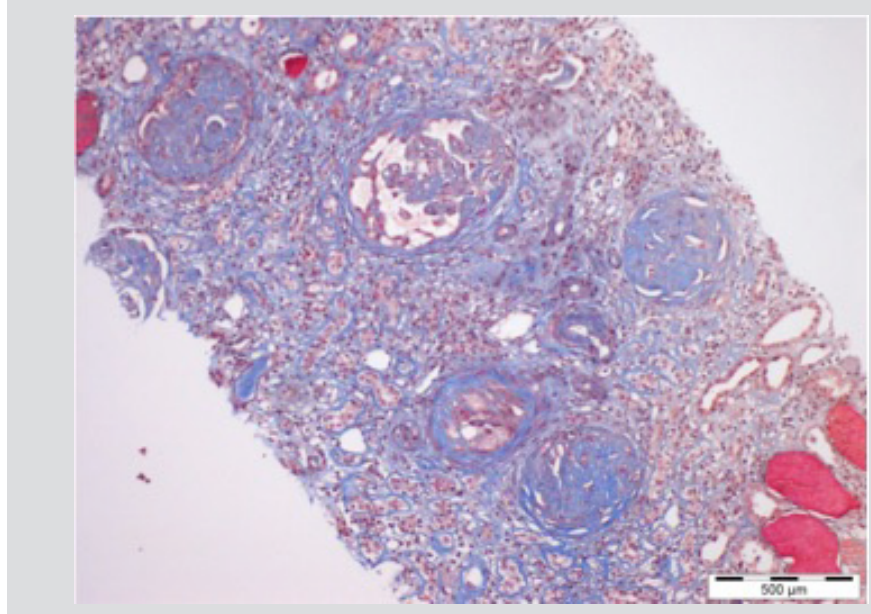

Figure 3. Congo red-positive glomerular deposits 


\section{DISCUSSION}

Amyloidosis in patients with primary SS is rare ${ }^{[1,8]}$. The risk of developing AA amyloidosis is related to continuous, subclinical inflammation and, consequently to the magnitude and persistence of elevated AA amyloid levels ${ }^{[1]}$. In the case we describe, unfortunately it was not possible to measure the serum amyloid A level. Related to this, monoclonal gammopathy is frequently found in SS patients, with monoclonal IgGK being the most frequent band type detected ${ }^{[10]}$.

Ooms et al. reported the first case of primary SS with secondary renal AA amyloidosis in a patient with long-standing primary SS who presented with acute renal failure and nephrotic syndrome. Renal biopsy revealed concomitant renal AA amyloidosis and chronic interstitial nephritis. The patient was treated with corticosteroids and achieved partial remission of the nephrotic syndrome and improvement in renal function. The authors assumed that the chronic interstitial nephritis itself, associated with persistent disease activity, stimulated the development of the renal AA amyloidosis. They argue that SS should be regarded as a predisposing condition for the development of renal AA amyloidosis. Therefore, renal biopsy is essential in patients with SS who develop renal insufficiency or nephrotic proteinuria ${ }^{[1]}$.

In cases where amyloidosis accompanies SS, amyloidosis is diagnosed 1-25 years after the development of inflammation, during the course of SS, although in some cases these conditions have been diagnosed simultaneously. This may be the result of delayed diagnosis of SS in patients who previously had only mild or no symptoms associated with the disease ${ }^{[8]}$, which seems to be the case in our patient.

Renal involvement in SS is generally pauci-symptomatic and therefore, serum and urinary analysis is required to detect and prevent endstage renal disease $\mathrm{e}^{[4]}$. Although it is a well-known complication of chronic inflammatory disease, few studies have evaluated the natural history, prognostic markers and treatment of AA amyloidosis ${ }^{[6]}$.

\section{CONCLUSION}

Renal AA amyloidosis is a rare condition in patients with primary SS. However, in patients with proteinuria and/or renal failure, it should be included in the differential diagnosis. Renal biopsy should be performed to establish a definitive diagnosis.

\section{REFERENCES}

1. Ooms V, Decupere M, Lerut E, Vanrenterghem Y, Kuypers DR. Secondary renal amyloidosis due to long-standing tubulointerstitial nephritis in a patient with Sjögren syndrome. Am J Kidney Dis 2005;46(5):e75-80.

2. Nikolova M, Ivanov G, Makova G. Sjogren's syndrome and the kidney. Arch Immunol Allergy 2018;1(2):20-24.

3. Evans R, Zdebik A, Ciurtin C, Walsh S. Renal involvement in primary Sjogren's syndrome. Rheumatology 2015;54:1541-1548.

4. François H, Mariette X. Renal involvement in primary Sjorgren's syndrome. Nat Rev Nephrol 2016;12(2):82-93.

5. Erdogmus S, Kendi Celebi Z, Akturk S, Kumru G, Duman N, Ates K, et al. Profile of renal AA amyloidosis in older and younger individuals: a single-centre experience. Amyloid 2018;25(2):115-119.

6. Yilmaz M, Unsal A, Sokmen M, Kaptanogullari OH, Alkim C, Kabukcuoglu F, et al. Renal involvement in AA amyloidosis: clinical outcomes and survival. Kidney Blood Press Res 2013;37(1):33-42.

7. Lachmann HJ, Goodman HJ, Gilbertson JA, Gallimore JR, Sabin CA, Gillmore JD, et al. Natural history and outcome in systemic AA amyloidosis. N Engl J Med 2007;356(23):23612371.

8. Kweon SM, Koh JH, Lee HN, Kim E, So MW, Shin HJ, et al. Primary Sjogren syndrome diagnosed simultaneously with localized amyloidosis of the lacrimal gland: a case report. Medicine (Baltimore) 2018;97(23):e11014.

9. Dember LM. Amyloidosis-associated kidney disease. J Am Soc Nephrol 2006;17(12):3458-3471.

10. Brito-Zerón P, Retamozo S, Gandía M, Akasbi M, Pérez-De-Lis M, Diaz-Lagares C, et al. Monoclonal gammopathy related to Sjögren syndrome: a key marker of disease prognosis and outcomes. J Autoimmun 2012;39(1-2):43-48. 